referring to a specialist diagnostic team was about 6 months. However, after a screening questionnaire had taken place, the time to referral was only around one month. We propose that screening is considered at an earlier opportunity; ideally during (or prior to) the first appointment with the CMHT in order to reduce the time before a referral to a specialist diagnostic team is made. This would enable treatment in a care pathway which incorporates the diagnosis of ASD at an earlier stage.

\section{Development and validation of a non-remission risk prediction model in First Episode Psychosis: An analysis of two longitudinal studies}

Samuel Leighton ${ }^{1 \star}$, Pavan Mallikarjun², Rajeev Krishnadas ${ }^{3}$, Jonathan Cavanagh ${ }^{1}$, Simon Rogers ${ }^{1}$, Rachel Upthegrove ${ }^{2}$, Max Birchwood ${ }^{4}$, Stephen Marwaha ${ }^{2}$, Ewout Steyerberg 5 , Georgios Gkoutos ${ }^{2}$, Matthew Broome ${ }^{2}$, Peter Liddle ${ }^{6}$, Linda Everard 7 , Swaran Singh ${ }^{4}$, Nicholas Freemantle ${ }^{8}$, David Fowler ${ }^{9}$, Peter Jones ${ }^{10}$, Vimal Sharma ${ }^{11}$, Robin Murray ${ }^{12}$, Til Wykes ${ }^{12}$, Richard Drake ${ }^{13}$, Iain Buchan ${ }^{14}$ and Shon Lewis ${ }^{13}$

${ }^{1}$ University of Glasgow; ${ }^{2}$ University of Birmingham; ${ }^{3}$ NHS Greater Glasgow \& Clyde; ${ }^{4}$ University of Warwick; ${ }^{5}$ University of Leiden;

${ }^{6}$ University of Nottingham; ${ }^{7}$ Neuropsychiatry - The Barberry Center; ${ }^{8}$ University College London; ${ }^{9}$ University of Sussex; ${ }^{10}$ University of Cambridge; ${ }^{11}$ University of Chester; ${ }^{12}$ Institute of Psychiatry, Psychology and Neuroscience, King's College London;

${ }^{13}$ University of Manchester and ${ }^{14}$ University of Liverpool ${ }^{*}$ Corresponding author.

\section{doi: 10.1192/bjo.2021.147}

Aims. Psychosis is a major mental illness with first onset in young adults. The prognosis is poor in around half of the people affected, and difficult to predict. The few tools available to predict prognosis have major weaknesses which limit their use in clinical practice. We aimed to develop and validate a risk prediction model of symptom non-remission in first-episode psychosis.

Method. Our development cohort consisted of 1027 patients with first-episode psychosis recruited between 2005 to 2010 from 14 early intervention services across the National Health Service in England. Our validation cohort consisted of 399 patients with first-episode psychosis recruited between 2006 to 2009 from a further 11 English early intervention services. The one-year non-remission rate was $52 \%$ and $54 \%$ in the development and validation cohorts, respectively. Multivariable logistic regression was used to develop a risk prediction model for non-remission, which was externally validated.

Result. The prediction model showed good discrimination (C-statistic of $0.74(0.72,0.76)$ and adequate calibration with intercept alpha of $0.13(0.03,0.23)$ and slope beta of $0.99(0.87,1.12)$. Our model improved the net-benefit by $16 \%$ at a risk threshold of $50 \%$, equivalent to 16 more detected non-remitted first-episode psychosis individuals per 100 without incorrectly classifying remitted cases.

Conclusion. Once prospectively validated, our first episode psychosis prediction model could help identify patients at increased risk of non-remission at initial clinical contact.

\section{Audit on resuscitation equipment in Carseview Centre (NHS Tayside)}

Thomas Leung*, Lina Zariddin and Emma Megoran

NHS Tayside

${ }^{\star}$ Corresponding author.

doi: 10.1192/bjo.2021.148
Aims. Psychiatric hospitals are well equipped to manage patients with complex psychiatric needs, however due to their community setting when a rare medical emergency occurs it is not unusual for a small delay whilst staff search for equipment on the ward or even go to other wards for equipment. The aim of this audit is to ensure that our psychiatric wards in Carseview Centre are well equipped to respond to patients becoming medically unwell and put our nurses and doctors in a position to safely stabilise the patient until furthur help arrives.

Method. We collected data from 3 inpatient adult wards, 1 intensive psychiatric care unit and 1 learning disability unit and compared their resuscitation trolley equipment with local NHS Tayside Emergency Equipment Protocol in January 2020. Following data collection we fed back to the wards about our results and discussions were held between doctors, charge nurses, pharmacists and resuscitation officers to determine whether missing equipment were neccesary in the community setting and to see if there were updates that required for our local protocol to better reflect current practices as it had not been reviewed since 2012. Following multiple meetings we amended our local protocol to better reflect what was. A list of recommendations was also made to improve patient safety.

We then collected data again in January 2021

Result. Folloing our first data collection we found that the resuscitation trolleys tended to not have ligature packs and masks were generally not by the oxygen cylinders. Hypoglycaemic dextrotablets were also not readily available. The Learning disability units also did not have an emergency resuscitation trolley.

Following our discussions and amendment of the protocol this was finalised in November 2020 and was dissemindated towards the wards and we waited 2 months for the changes to take effects and recollected our data. There continued to be equipment that was incomplete/missing on each individual ward, but none that were consistent throughout the whole hospital site. All the recommendations that were made for the 1st data collection had been done.

Conclusion. Overall we felt that the emergency trolleys were better equipped in line with the updated protocol compared to the previous audit cycle. The overall pattern of missing equipment was inconsistent and the recommendation was for staff to copmlete checks to address missing/incomplete items when found. Our local protocol also recommends that all ward should stock 'additional items' (nebuliser masks and non-rebreather masks), which majority had however were difficult to locate, which could delay patient care.

We will continue to repeat data collection cycles and feedback to our wards to ensure patient safety is not compromised.

\section{Psychopathology and cognitive deficits in young people exposed to complex trauma}

Stephanie J Lewis ${ }^{1 *}$, Karestan C Koenen ${ }^{2}$, Antony Ambler ${ }^{3}$, Louise Arseneault ${ }^{3}$, Avshalom Caspi ${ }^{4}$, Helen L Fisher ${ }^{3}$, Terrie E Moffitt ${ }^{4}$ and Andrea Danese ${ }^{1}$

${ }^{1}$ Institute of Psychiatry, Psychology \& Neuroscience, King's College London, South London and Maudsley NHS Foundation Trust; ${ }^{2}$ Harvard TH Chan School of Public Health; ${ }^{3}$ Institute of Psychiatry, Psychology and Neuroscience, King's College London and ${ }^{4}$ Duke University, Institute of Psychiatry, Psychology and Neuroscience, King's College London

${ }^{*}$ Corresponding author.

doi: 10.1192/bjo.2021.149

Aims. Complex traumas are traumatic experiences that involve multiple interpersonal threats during childhood or adolescence, 